\title{
Reciclando la clasificación sinóptica de Font: reconstrucción con ERA40 y agrupamiento PCA
}

\author{
Carlos Santos Burguete ${ }^{1}$ (csantosb@aemet.es) \\ Álvaro Subías Díaz-Blanco² (asubiasd@aemet.es) \\ Alejandro Roa Alonso² (aroaa@aemet.es)
}

${ }^{1}$ AEMET / Dirección de Producción e Infraestructuras / Centro Nacional de Predicción

${ }^{2}$ AEMET / Dirección de Producción e Infraestructuras / Área de Técnicas y Aplicaciones de Predicción

\begin{abstract}
RESUMEN
Las clasificaciones sinópticas ayudan a entender la variabilidad y complejidad de los llamados patrones meteorológicos, o situaciones, a escala sinóptica. Hay una gradación de clasificaciones, desde las subjetivas, con caracterizaciones conceptuales y diagnósticas, hasta las clasificaciones objetivas, basadas en soporte numérico y con un amplio abanico de familias de algoritmos asociados, pasando por clasificaciones intermedias que tienen carácter mixto. En este estudio se revisan la clasificación objetiva de Ribalaygua y Borén (1995) y la clasificación subjetiva de Font (2000). Esta última propone 23 patrones sinópticos, ilustrados con situaciones de 23 fechas concretas, en general de la década de 1970-1980. Se recuperan los reanálisis ERA40 (Uppala et al., 2005) del European Centre for Medium-range Weather Forecasts (ECMWF) correspondientes a esas $23 \mathrm{fe}$ chas y se dibujan los campos básicos con las herramientas actuales y estilo de visualización de predicción operativa. Se compara así el querer de la mano con el querer de la máquina. Se aplica, además, a estas 23 situaciones un algoritmo de agrupamiento «análisis de componentes principales» (PCA por sus siglas en inglés; JoLLIFFE, 1986) similar al vigente en AEMET para el sistema de predicción por conjuntos del ECMWF, con el propósito de aprender sobre la naturaleza de la variabilidad y diversidad de estos patrones. Se trata, pues, de reciclar un conocimiento muy conceptual y valioso, el de Font, conectándolo con el potencial de las herramientas numéricas actuales para, en definitiva, ver qué podemos aprender.
\end{abstract}

PALABRAS CLAVE: clasificaciones sinópticas; patrones sinópticos; predicción numérica; predicción operativa; modelos conceptuales; climatología sinóptica.

\section{INTRODUCCIÓN}

Los fenómenos meteorológicos se presentan en un amplio abanico de escalas espaciales y sus correspondientes escalas temporales. De forma sencilla, las escalas planetaria, sinóptica, mesoescala y microescala abarcan fenómenos desde los $10000 \mathrm{~km}$ hasta los centímetros y, correspondientemente, desde meses o semanas hasta segundos. La escala sinóptica alberga fenómenos del orden de 1000 km y horas-días de duración. Los más conocidos son las vaguadas y dorsales en niveles medio-altos y los anticiclones y borrascas en niveles bajos de la troposfera. La llamada climatología sinóptica (FonT, 2000) caracteriza el clima en dicha escala basándose en los diversos factores que lo configuran, poniendo especial atención a los diversos patrones de circulación atmosférica, tarea que, por la complejidad y cantidad de los mismos, requiere un esfuerzo importante de síntesis. 
Estas clasificaciones de patrones sinópticos espaciales resultan útiles como herramienta tanto analítica y predictiva como didáctica y de comunicación, tanto para el tiempo como para el clima. Existen, por un lado, clasificaciones subjetivas (LiNÉs, 1981; Font, 2000; SÁNCHEZ RodRíGUEZ; 1993) basadas tradicionalmente en el conocimiento y la experiencia de profesionales de la predicción o la climatología, de carácter fundamentalmente cualitativo y con fuerte conexión con los efectos que produce cada patrón sobre el tiempo sensible. Por otro lado, desde la aparición de las técnicas de clustering o agrupamiento (HARTIGAN y HARTIGAN, 1975) con soporte computacional, existen clasificaciones objetivas basadas en algoritmos de similitud morfológica (Calvo, 1993; Petisco y Martín, 1995; Ribalaygua y Borén, 1995). Ambos enfoques, subjetivo y objetivo, son importantes y aportan conocimiento y utilidad complementarios. Ofrecemos aquí un ejemplo particular de cada enfoque, suficiente para entender las bases, resultados y conclusiones de ambos métodos.

En la sección 2 mostramos las clasificaciones subjetivas, dando el ejemplo de la clasificación de FonT (2000). En la sección 3 revisamos los fundamentos de las clasificaciones objetivas, ilustrando con el ejemplo de la clasificación de Ribalaygua y Borén (1995). En la sección 4 se recicla la clasificación de Font con una presentación moderna y se aplica una técnica de agrupación de los patrones de Font. Siguen las conclusiones y las referencias.

\section{CLASIFICACIONES SUBJETIVAS}

\subsection{Masas de aire}

En la caracterización de los patrones sinópticos es crucial el concepto de masa de aire. Una región del planeta suficientemente grande y meteorológicamente homogénea, que llamaremos región fuente o manantial, puede conferir a la masa de aire que soporta propiedades particulares de opacidad, humedad, temperatura, etc. Esta masa de aire viajará y conservará estas propiedades un tiempo, durante el cual podrá afectar a otras regiones. La tabla 1 (FonT, 2000) ofrece un detalle de aquellas masas de aire que afectan principalmente a la península ibérica. El indicativo de cada masa de aire se compone de una letra minúscula que indica su origen (m marítimo, c continental) y una mayúscula que denota su carácter (T tropical, P polar, Aártica).

\begin{tabular}{|c|c|c|c|c|}
\hline \multicolumn{2}{|c|}{ Naturaleza } & \multirow{2}{*}{$\begin{array}{l}\text { Origen } \\
\text { Océano Ártico } \\
\text { Groenlandia, } \\
\text { N Canadá }\end{array}$} & \multirow{2}{*}{\begin{tabular}{l|} 
Indicativo \\
$\mathrm{mA}$ \\
$\mathrm{mP}$
\end{tabular}} & \multirow{2}{*}{$\begin{array}{l}\text { Meses de más frecuencia } \\
\text { Invierno y abril } \\
\text { Invierno y ocasionalmente resto } \\
\text { del año }\end{array}$} \\
\hline Frías & Marítimas & & & \\
\hline & Continentales & Rusia, Siberia & $\mathrm{CP}$ & Febrero, diciembre, enero \\
\hline \multirow[t]{2}{*}{ Cálidas } & Marítimas & $\begin{array}{l}\text { Atlántico subtropical } \\
\text { Atlántico tropical }\end{array}$ & $\begin{array}{l}\mathrm{mT} \text { (sub) } \\
\mathrm{mT}\end{array}$ & $\begin{array}{l}\text { Verano y ocasionalmente resto } \\
\text { del año } \\
\text { Otoño, invierno y ocasionalmente } \\
\text { primavera }\end{array}$ \\
\hline & Continentales & N África & cT & \\
\hline
\end{tabular}

Tabla 1. Principales masas de aire que afectan a la península ibérica.

La figura 1.a (FONT, 2000), ilustra las trayectorias de las masas de aire que principalmente afectan a la península ibérica (PI). Esta, a su vez, puede actuar sobre las masas de aire, modificando sus propiedades, si estas permanecen el tiempo suficiente sobre la misma. Este aspecto se aborda más adelante. 
a)

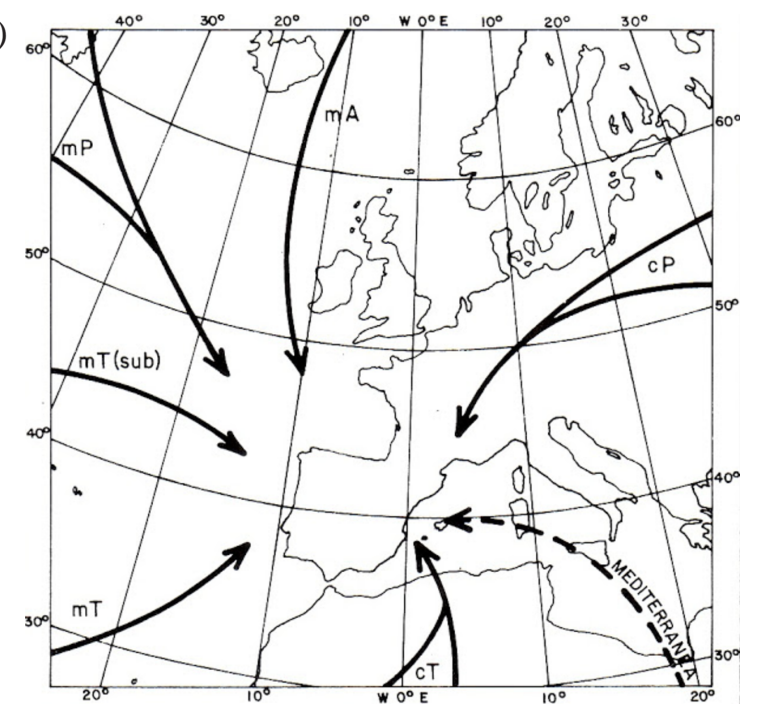

b)

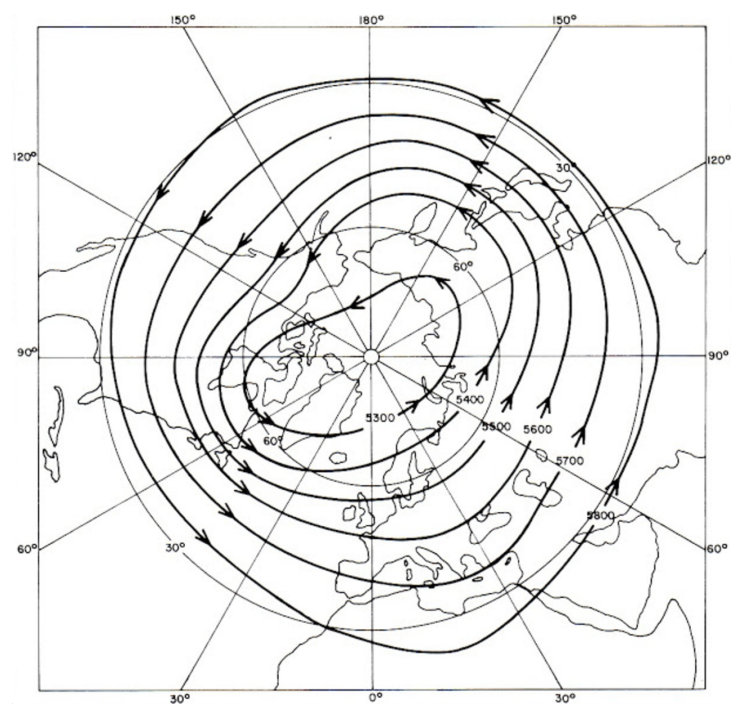

Figura 1. a) Trayectorias de las masas de aire que llegan a la PI. b) Media anual de la altura geopotencial en $500 \mathrm{hPa}$, estructura planetaria que llamamos vórtice circumpolar (Font, 2000).

\subsection{Vórtice circumpolar}

La configuración de flujo planetario y sinóptico en niveles medio-altos de la troposfera (500 hPa) y sus variaciones son cruciales para la climatología sinóptica. En latitudes medias (aproximadamente $30^{\circ}-60^{\circ}$ ) predominan los vientos de componente oeste en casi todos los niveles por encima de $3000 \mathrm{~m}$, lo que viene a llamarse vórtice circumpolar, ilustrado en la figura 1.b.

a)

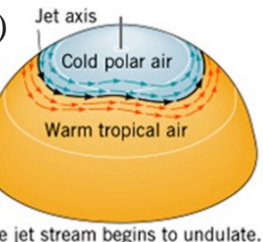

The jet stream begins to undulate.

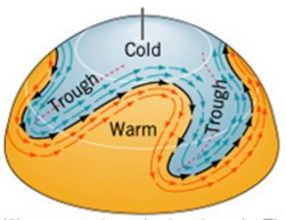

Waves are strongly developed. The cold air occupies troughs of low pressura
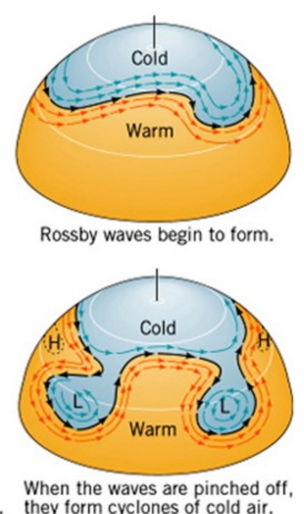

b)

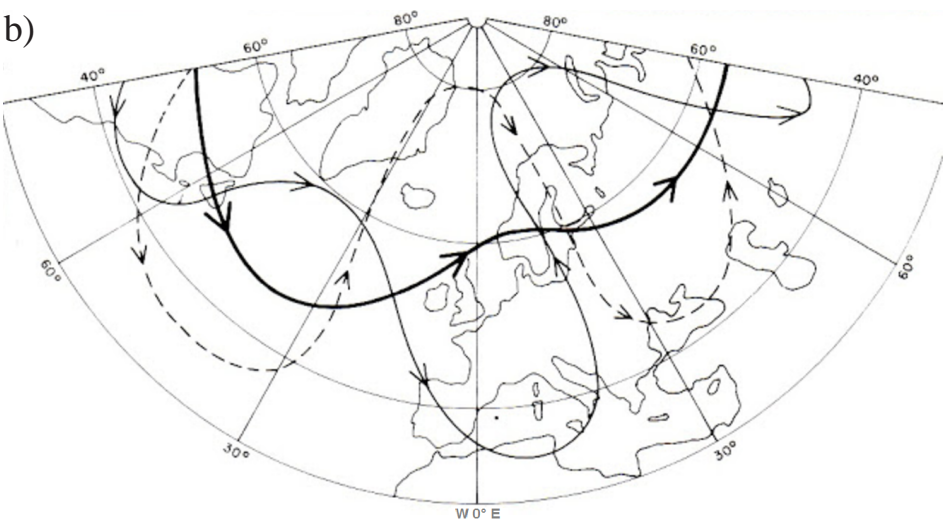

Figura 2. Flujos zonal y ondulado. a) Fluctuaciones cíclicas no periódicas del flujo del oeste entre flujo zonal y flujo meridiano (STRAHLER y STRAHLER, 2007). b) Flujo zonal en línea gruesa, flujos meridianos en línea fina y línea discontinua (Font, 2000).

Las intensidades máximas (típicamente $300 \mathrm{~km} / \mathrm{h}$ ) se encuentran en las inmediaciones de la tropopausa (altitudes de 8-12 km) en la denominada corriente en chorro (jet stream). El chorro, al no tener posiciones fijas en el planeta, no aparece en los mapas climatológicos, en los que se promedia en el tiempo, aunque sí aparece en los mapas del tiempo. El vórtice circumpolar es más intenso en invierno que en verano. Además, sufre fluctuaciones no periódicas (esencialmente caóticas) entre lo que se denomina flujo zonal, siguiendo aproximadamente los paralelos y flujo meridiano u ondulado con componentes meridionales (figura 2.a).

En el flujo ondulado se desarrollan bajas frías (así como altas cálidas) en altura a latitudes más bajas que las borrascas del frente polar. Un caso particular muy importante en España es el fenómeno denominado depresión aislada en niveles altos (dana) que favorece precipitaciones intensas en el Mediterráneo peninsular 
(fenómeno denominado popular y equivocadamente gota fría). Estas bajas pueden o no tener reflejo en superficie y, en caso de tenerlo, la parte superior suele estar retrasada (posición más al oeste) que la de superficie. Así mismo, el vórtice circumpolar desarrolla de forma no periódica contracciones y expansiones que desplazan los máximos de viento al norte o al sur. De forma muy general (ilustrada en la figura 2.b), podemos decir que la circulación zonal en altura va asociada a pequeñas borrascas de paso rápido, sobre la PI si el eje está relativamente al sur, o más al norte de la PI, dejando entonces tiempo seco. La circulación meridiana, según la posición relativa de los meandros, puede traer aire frío en altura que, en conjunción con el Mediterráneo relativamente cálido, genera condiciones muy inestables, o bien puede traer aire subtropical cálido y más seco.

Denominamos tipo de tiempo a la distribución particular de los sistemas de presión y de las masas de aire sobre una región geográfica especificada, asociados con típicas características generales del tiempo atmosférico. La clasificación sinóptica de tipos de tiempo sobre la PI es compleja y, en estas clasificaciones tradicionales, subjetiva. Un ejemplo famoso es la clasificación de FonT (2000).

\subsection{Clasificación subjetiva de FonT (2000)}

Font utiliza dos factores: la contracción-expansión del vórtice circumpolar y, en el caso normal de la misma, el tipo de circulación —zonal, meridiana odepresión fríadistinguiendo además, cuando se da el caso, si es predominantemente invernal o más bien estival (tabla 2).

\begin{tabular}{|l|l|}
\hline Circulación en altura del oeste \\
\hline \multirow{3}{*}{$\begin{array}{l}\text { Latitud } \\
\text { I Latitudes normales }\end{array}$} & Forma \\
\cline { 2 - 2 } & Z Zonal \\
\cline { 2 - 2 } & M Meridiana \\
\cline { 2 - 2 } & D Depresión fría claramente separada del vórtice \\
\hline II Latitudes altas & \\
\hline III Latitudes bajas & \\
\hline
\end{tabular}

Tabla 2. Factores para la clasificación sinóptica de FonT (2000).

a)
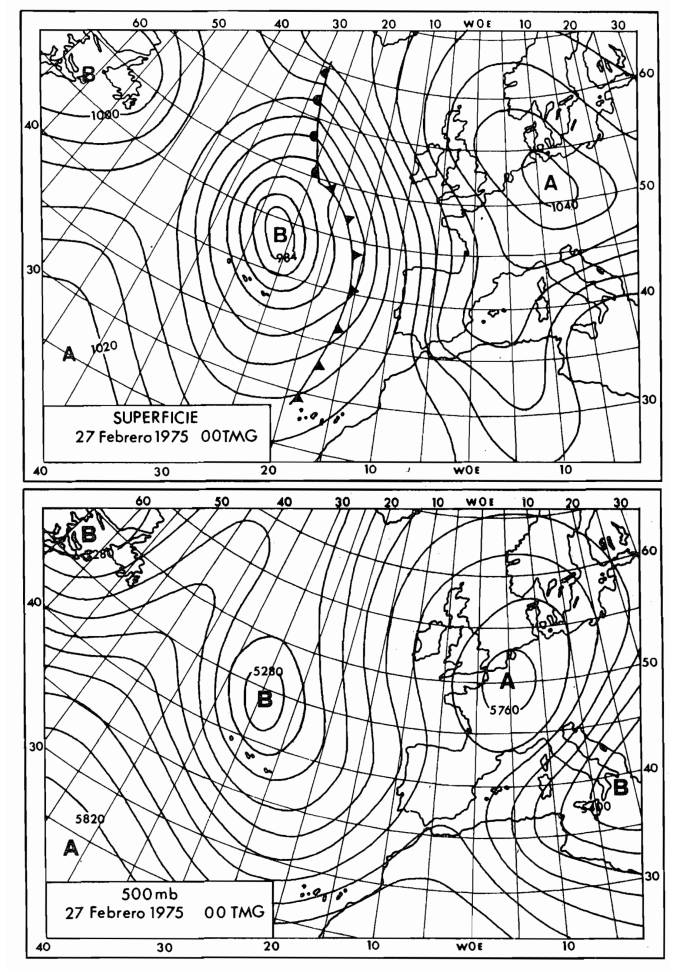

Fig. 68 Tipo de tiempo n. ${ }^{\circ}$ II..M.i.- Anticiclón centroeuropeo. b)

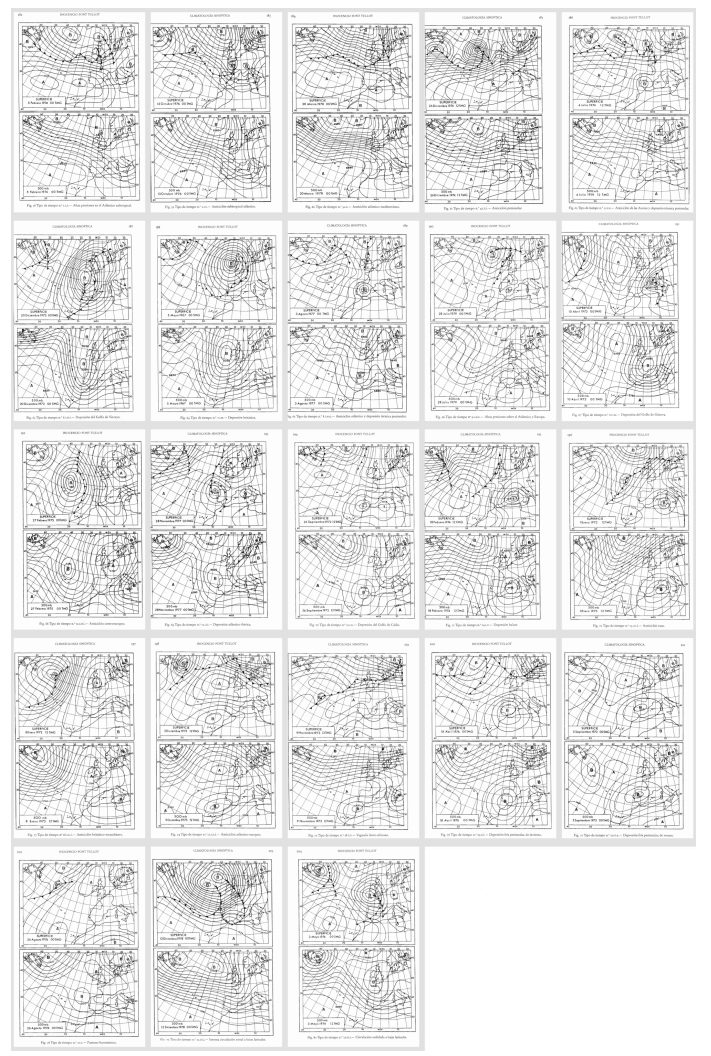

Figura 3. a) Situación del 27 de febrero de 1975 tipo Font «Anticiclón centroeuropeo», con la reproducción original de los mapas delineados. b) Mosaico con los 23 patrones originales de Font. 
Atendiendo a estos factores combinados, Font encuentra 23 grupos subjetivos que representan tipos de tiempo que mantienen sus rasgos generales tres días o más, con periodos de transición muy difícilmente caracterizables. En FonT (2000) se presentan estos tipos de tiempo mostrando los mapas delineados manualmente (el querer de la mano), correspondiendo cada caso a una fecha de la década 1970-1980 aproximadamente. Se muestra un ejemplo en la figura 3.a y una panorámica de los 23 patrones en la figura 3.b.

\section{CLASIFICACIONES OBJETIVAS}

\subsection{Fundamentos}

Desde el surgimiento de los modelos atmosféricos de predicción, las predicciones existen en forma de números, cubriendo habitualmente un dominio espacial no solo en superficie, sino también en una serie de niveles verticales que suelen abarcar la troposfera (y últimamente parte de la estratosfera), con una densidad o resolución que depende de la escala. En la sinóptica las resoluciones actuales (2018) rondan los 10-30km.

¿Cómo funcionan las clasificaciones basadas en técnicas de agrupamiento con soporte computacional? En los algoritmos más sencillos, para clasificar se agrupan las situaciones según un criterio numérico de similitud. Dado que cada situación es un campo numérico, la similitud entre situaciones puede calcularse, por ejemplo, como la distancia euclídea (o alguna otra medida de distancia) entre dos vectores. Cuando esa distancia está por debajo de un umbral prefijado, agrupamos las dos situaciones formando un grupo. Así sucesivamente hasta haber recorrido todas las comparaciones posibles. Este algoritmo básico puede mejorarse y hacerse más eficaz y sutil, incluyendo varianza explicada, número de grupos, etc. En la actualidad el agrupamiento conforma toda una disciplina científica y técnica y existe una gran variedad de familias de algoritmos. Son populares, en la comunidad de clima, algoritmos como el análisis de componentes principales (PCA por sus siglas en inglés; JOLLIFFE, 1986), etc.

Según la capacidad computacional de que se disponga, las clasificaciones pueden ser más o menos exhaustivas. Con poca capacidad, se reduce la agrupación a la similitud en un solo campo. Desde un punto de vista práctico, para la escala sinóptica se escoge a menudo un campo de naturaleza dinámica, como es la altura geopotencial en niveles medio-altos o la presión reducida al nivel del mar. Estos campos presentan variabilidades relativamente suaves en la escala sinóptica, lo que facilita la eficacia y utilidad del algoritmo. Campos de tiempo sensible como son la precipitación o la temperatura en superficie son mucho más ruidosos, por lo que las clasificaciones son, en principio, más complejas y requieren normalmente consideraciones adicionales. Con más capacidad computacional puede trabajarse con varios campos simultáneamente lo que enriquece, como es natural, la clasificación pero a la vez la hace más compleja: más grupos y más características a describir. En cualquier caso, se necesita una base de datos adecuada en tamaño, así como fiabilidad y representatividad de los mismos.

\subsection{Clasificación objetiva de Ribalaygua y Borén (1995)}

Esta clasificación (RIBALAYGUA y Borén, 1995), aunque no es de las más modernas, tiene un interés especial porque es consistente con clasificaciones subjetivas tradicionales, puede interpretarse bien en términos diagnósticos (aquellos expresados en lenguaje meteorológico) y, además, la agrupación se basa en una variable de tiempo sensible como es la precipitación. El procedimiento se ilustra en la figura 4.a. En un primer paso se aplica el algoritmo de agrupamiento al campo de precipitación diaria acumulada. Una vez que se tienen los grupos, se caracteriza cada grupo sinópticamente por los campos de altura geopotencial en $500 \mathrm{hPa}(\mathrm{Z} 500)$ y presión reducida al nivel medio del mar (MSLP), promediados a partir de los elementos de cada grupo.

Hay varias ventajas en este procedimiento: el campo de precipitación (PCP), si no se llega a resoluciones altas, y debido a la influencia compartimentadora de la orografía, suele presentar un número limitado de patrones. Así mismo, las clases sinópticas resultantes tendrán alta capacidad predictiva, dado que la clasificación 
a)

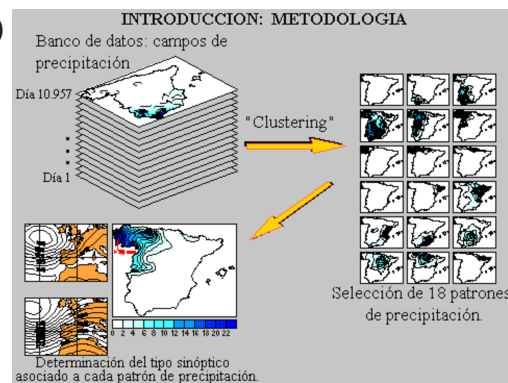

b)

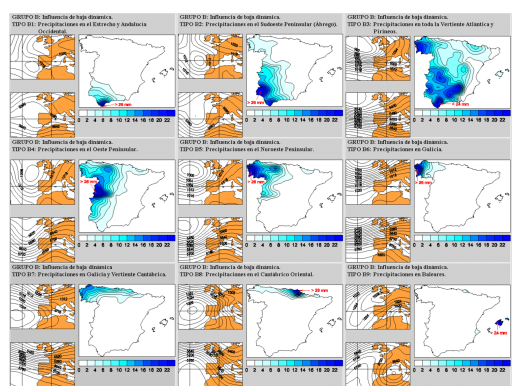

c)

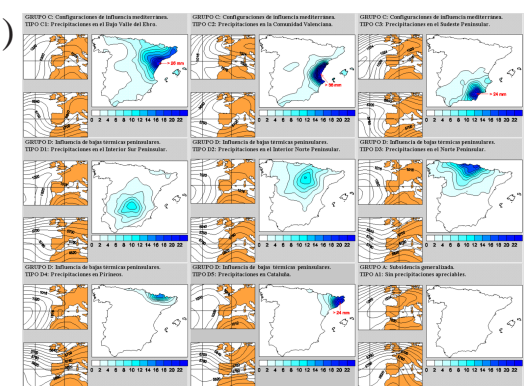

Figura 4. Agrupación de situaciones meteorológicas de RiBALAYGUa y Borén (1995) que produce 18 patrones meteorológicos en 4 grupos (véase texto). a) Esquema del procedimiento. b) Grupo B con 9 patrones.

c) Grupos C, D y A con 9 patrones en total. Fuente: AEMET.

se ha basado en el efecto en superficie. La base de datos abarca el periodo 1961-1990 con PCP de 10957 días, en un dominio sobre PI y Baleares, con más de 3000 estaciones. Las configuraciones sinópticas se representan en una malla regular con resolución de $3^{\circ} \times 4^{\circ}$.

Como resultado, se obtienen 18 patrones de precipitación y sus 18 configuraciones sinópticas asociadas. La relación entre la configuración sinóptica de cada grupo y el patrón de precipitación asociado puede interpretarse en términos de modelos conceptuales meteorológicos de precipitación descritos, por su interés, en el siguiente apartado. Los 18 patrones se pueden ordenar conceptualmente según la naturaleza de la configuración y según la zona de descarga de precipitaciones. Tendremos así los grupos A, B, C y D, presentados resumidamente en la tabla 3 y las figuras 4.b (Grupo B) y 4.c (grupos C, D y A). En conjunto, los 18 patrones quedan ordenados de forma que la trayectoria de las sucesivas zonas de precipitación describe una especie de espiral, que comienza en el área del Estrecho con el tipo B1 y termina en Cataluña con el tipo D5.

\begin{tabular}{|c|c|c|}
\hline Grupo & Patrón sinóptico & Precipitaciones \\
\hline $\begin{array}{l}\text { Grupo A: } \\
\text { subsidencia } \\
\text { generalizada }\end{array}$ & $\begin{array}{l}\text { Predominan los descensos (subsidencias) de aire sobre la } \\
\text { PI y Baleares, impidiendo la precipitación. Aparecen con } \\
\text { frecuencia similar en todas las estaciones del año. }\end{array}$ & $\begin{array}{l}\text { Sin precipitaciones } \\
\text { apreciables. }\end{array}$ \\
\hline $\begin{array}{l}\text { Grupo B: } \\
\text { baja } \\
\text { dinámica }\end{array}$ & $\begin{array}{l}\text { Predominan los forzamientos frontal y orográfico. La } \\
\text { presencia de una baja o borrasca de origen dinámico } \\
\text { genera frentes más o menos activos o líneas de } \\
\text { inestabilidad. Los flujos superficiales están bien definidos, } \\
\text { con precipitaciones muy influidas por la orografía. Z500 } \\
\text { tiene una importancia secundaria. Frecuencia mínima en } \\
\text { verano y superior en invierno, cuando el frente polar y el } \\
\text { cinturón de borrascas extratropicales asociado bajan a } \\
\text { nuestras latitudes. }\end{array}$ & $\begin{array}{l}\text { En las vertientes atlántica y } \\
\text { cantábrica, desplazándose la } \\
\text { zona de precipitación } \\
\text { máxima en el sentido de las } \\
\text { agujas del reloj, desde el área } \\
\text { del Estrecho (tipo B1), al } \\
\text { Cantábrico oriental (tipo B8). } \\
\text { El tipo B9 corresponde a } \\
\text { precipitaciones en Baleares. }\end{array}$ \\
\hline $\begin{array}{l}\text { Grupo C: } \\
\text { advección } \\
\text { mediterránea }\end{array}$ & $\begin{array}{l}\text { Predominio de vaguadas, bajas o depresiones aisladas en } \\
\text { niveles altos (danas) en niveles medios, acompañadas a } \\
\text { menudo por embolsamientos fríos. Así mismo, flujo } \\
\text { superficial de componente este (levante), húmedo y cálido. } \\
\text { Los forzamientos dinámico y orográfico favorecen los } \\
\text { ascensos. Dan lugar a precipitaciones convectivas de } \\
\text { distintas escalas, desde células aisladas hasta complejos } \\
\text { convectivos de mesoescala. En otoño la advección en } \\
\text { superficie es cálida además de húmeda, aumentando la } \\
\text { inestabilidad y por ende la intensidad y escala de los } \\
\text { fenómenos. Frecuencia máxima en primavera y otoño. }\end{array}$ & $\begin{array}{l}\text { En las vertientes } \\
\text { mediterráneas, } \\
\text { comenzando en el Bajo } \\
\text { Ebro (tipo C1) y } \\
\text { terminando en el sudeste } \\
\text { peninsular (tipo C3). Se } \\
\text { continúa con el mismo } \\
\text { criterio de desplazamiento. }\end{array}$ \\
\hline $\begin{array}{l}\text { Grupo D: } \\
\text { bajas } \\
\text { térmicas } \\
\text { peninsulares }\end{array}$ & $\begin{array}{l}\text { En niveles medio-altos vaguadas o borrascas móviles que dan } \\
\text { forzamientos dinámicos. Flujo superficial poco definido y, en } \\
\text { general, bajas térmicas en el interior peninsular. Se favorecen } \\
\text { los forzamientos térmicos e, indirectamente, dinámicos, con } \\
\text { flujos sobre cordilleras o flujos convergentes. La precipitación } \\
\text { es convectiva, dependiendo su organización e intensidad de la } \\
\text { estabilidad y la humedad en la atmósfera. Frecuencia superior } \\
\text { en verano. }\end{array}$ & $\begin{array}{l}\text { Concentradas en el interior } \\
\text { peninsular, desplazándose } \\
\text { la zona de precipitación } \\
\text { máxima de la Meseta sur } \\
\text { (tipo D1) a Cataluña } \\
\text { (tipo D5), también en } \\
\text { sentido horario. }\end{array}$ \\
\hline
\end{tabular}

Tabla 3. Clasificación de RiBalaygua y Borén (1995). 


\subsection{Modelos conceptuales de precipitación}

De forma simplificada, los fenómenos de precipitación se producen por ascenso de masas de aire húmedo. Al ascender, el aire se enfría, alcanzando la saturación, favoreciendo así la condensación en gotitas de nube e, indirectamente, gotas de agua. Los factores principales que intervienen en este proceso son:

- Forzamientos que influyen en la masa de aire para ascender, de varios tipos: orográfico, térmico y dinámico. El dinámico se asume como cuasigeostrófico (HolTon, 2012), e incluye los forzamientos frontales.

- Estabilidad atmosférica. Cuanto más estable la atmósfera, más dificultad tendrá la masa de aire en ascender. El perfil vertical de estabilidad depende de la densidad del aire que, a su vez, depende de las distribuciones verticales de temperatura y humedad.

- Contenido de humedad. La humedad es ingrediente básico en los procesos de condensación y su contenido, absoluto o relativo según las circunstancias, es relevante.

La caracterización sinóptica de RiBALAYGUA y BoRÉN (1995) con Z500 y MSLPes tradicional y sencilla y, aunque no informa directamente sobre temperatura y humedad, parámetros importantes en los procesos de precipitación, sí que pueden inferirse cualitativamente algunos aspectos:

- Los flujos de procedencia marina contienen mucha humedad.

- En vaguadas y borrascas las temperaturas en niveles medios tienden a ser relativamente bajas, pudiéndose inferir muy poca estabilidad.

- En el interior peninsular en clases con baja térmica las temperaturas en superficie son siempre elevadas, pudiéndose inferir de nuevo muy poca estabilidad.

\section{RECICLANDO LA CLASIFICACIÓN DE FONT (2000)}

\subsection{Técnica de presentación visual}

Para enriquecer la visualización, contrastar y reciclar el trabajo de FonT (2000) se han recuperado datos del reanálisis ERA40 del European Centre for Medium-range Weather Forecasts, ECMWF (UPPALA et al., 2005) correspondientes a las 23 fechas de los 23 tipos de Font. Se han reconstruido los mapas mediante la aplicación metview, específica del ECMWF para procesado y visualización de datos meteorológicos, siguiendo las pautas actuales (2018) en la intranet de AEMET, página del Área de Técnicas y Aplicaciones de Predicción (ATAP) de Visualización de Información Meteorológica (VIM), denominada coloquialmente Página de Feliciano en honor a su creador, FELICIANO JIMÉNEZ, anteriormente meteorólogo del ATAP. Como ejemplo, en la figura 5 se muestra uno de los casos de Font con la reproducción original de los mapas delineados (querer de la mano, figura 5.a) y la reconstrucción actual con los datos de ERA40 (querer de la máquina, figura 5.b). Podemos anticipar que, en primer lugar, el parecido es notable, como no podía ser de otra manera, subrayando así la capacidad profesional de los delineantes de la época. Podemos resaltar, además, algunos aspectos diferenciales. Para empezar, en los mapas manuales dibujaban frentes mientras que en el automático hemos dibujado estructuras nubosas. Es también notable lo bien perfilados que estaban los frentes, no disponiendo de imágenes de satélite, aunque en algunos patrones están mejor perfilados que en otros. Por otro lado, puede percibirse en el ploteo automático que se presentan algunas estructuras de menor escala que en el ploteo manual no aparecían, como por ejemplo un pequeño centro de baja presión en Argelia. Esta característica, en principio deseable por resaltar características mesoescalares, adolece sin embargo de cierta sensibilidad con los detalles del algoritmo de ploteo: por ejemplo, si ploteamos la presión cada hPa aparecen más estructuras, o estructuras diferentes, que si ploteamos cada 2 o cada $4 \mathrm{hPa}$; asimismo, el algoritmo de interpolación puede influir, a veces críticamente, en las estructuras emergentes. 
a) ${ }^{19}$
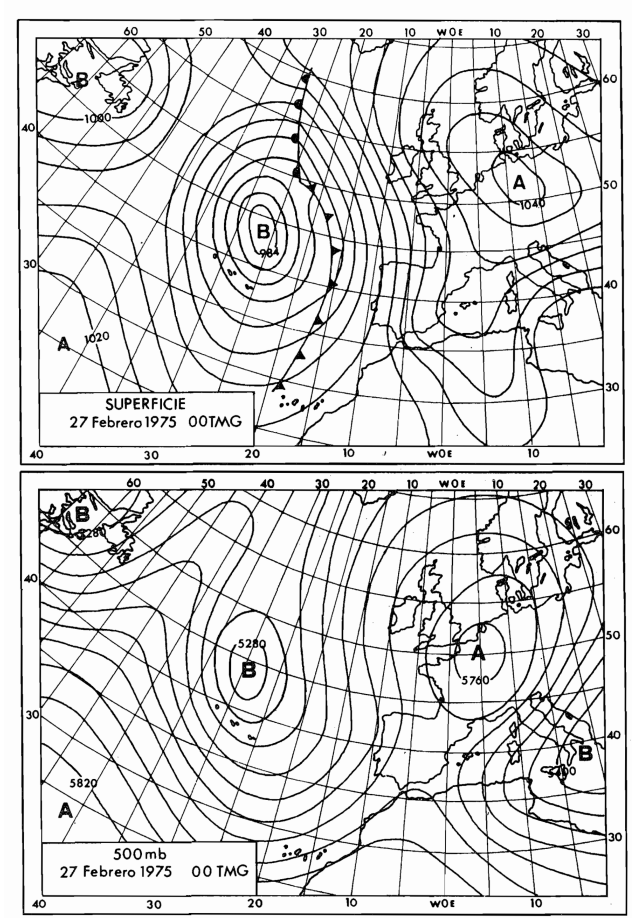

Fig. 68 Tipo de tiempo n. ${ }^{\circ}$ I...M.M.i.- Anticiclón centroeuropeo. b) Font 11: 1975-02-27 00 UTC Presion reducida al nivel del mar y cobertura nubosa

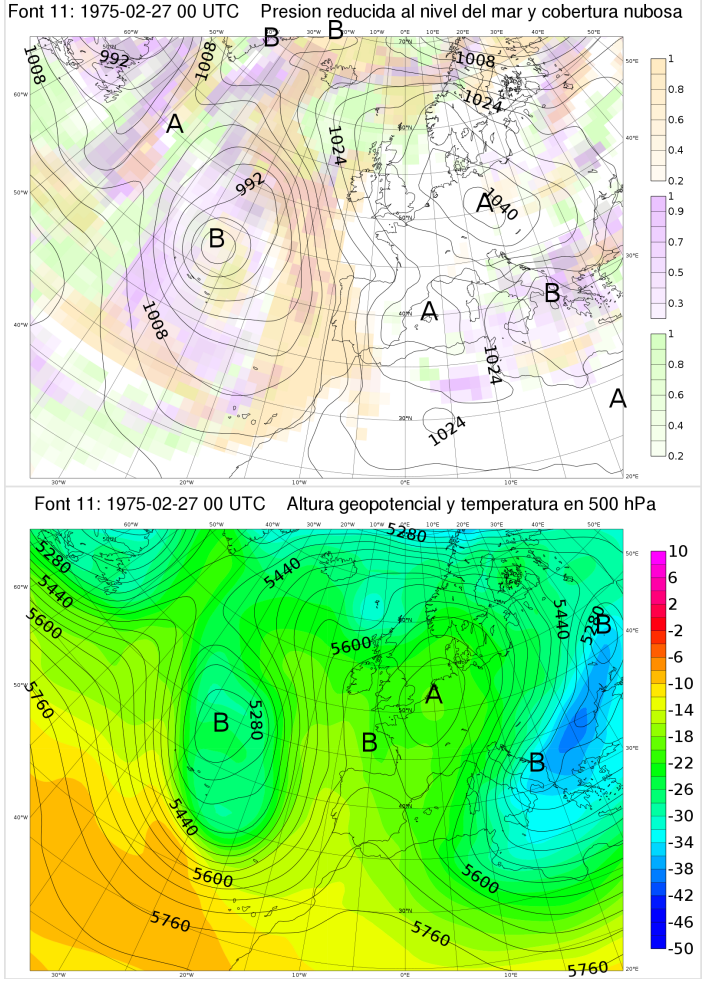

Figura 5. Situación del 27 de febrero de 1975 tipo 11 de Font «Anticiclón centroeuropeo», contrastando:

a) la reproducción original de los mapas delineados y b) la reconstrucción actual con los datos de ERA40 y la aplicación metview.

\subsection{Los 23 patrones de Font (2000)}

Puede encontrarse una descripción detallada de los 23 patrones, tipos o situaciones meteorológicas en FonT (2000). Se presentarán, de forma relativamente completa, en una nota técnica que AEMET tiene programado publicar en 2019. FONT (2000) sintetiza para cada patrón los siguientes elementos:

- Mapa en superficie: presión reducida al nivel del mar (isobaras) y cobertura nubosa (altas en rosa, medias en azul y bajas en verde).

- Mapa en altura: altura geopotencial (isohipsas) y temperatura (color) en $500 \mathrm{hPa}$.

- Número y nombre Font en negrita.

- Número, indicativo (véase tabla \{FACTORES FONT\}), masas de aire predominantes, meses de presencia (predominantes en tamaño superior, más frecuentes en negrita).

- Descripción meteorológica en altura (500).

- Descripción meteorológica en superficie (SFC).

- Duración: duración aproximada.

- Temperaturas $(\mathrm{T})$.

- Precipitaciones (PCP).

En la figura 6 se presentan los 23 patrones de Font, en su versión original (figura 6.a) y con la visualización actual (figura 6.b). 


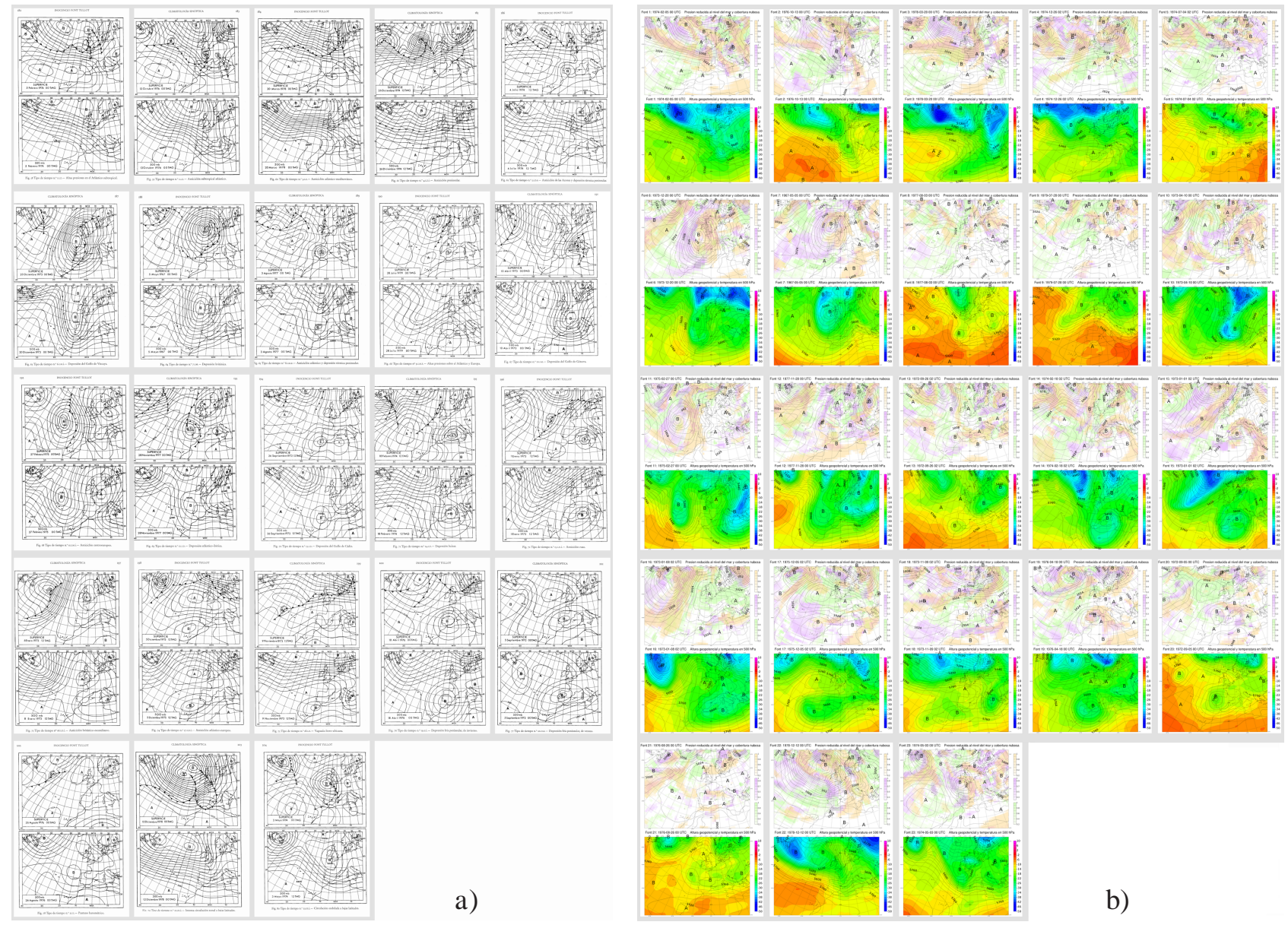

Figura 6. Los 23 patrones de Font. a) Reproducción original de los mapas delineados. b) Reconstrucción actual con los datos de ERA40 y la aplicación metview.

\subsection{Agrupamiento de los patrones}

Se ha realizado una primera aproximación, agrupando los 23 patrones mediante la técnica estadística de análisis de componentes principales (PCA por sus siglas en inglés, JOLLIFFE, 1986). Una técnica similar se utiliza en AEMET para agrupar diariamente la salida del sistema de predicción por conjuntos del ECMWF, denominado ENS - denominado coloquialmente EPS en AEMET porque en sus albores era el único EPS en la casa-. Los objetivos son, por un lado, explorar una posible simplificación de los 23 patrones; por otro, profundizar en el entendimiento de los mismos. Se ha aplicado la técnica PCA a los campos de altura geopotencial en $500 \mathrm{hPa}$ (Z500) de los 23 patrones de Font, para ceñirnos en principio a un solo parámetro. Estudios posteriores podrán ampliar ese abanico, por ejemplo usando también la presión reducida al nivel medio del mar. La media y desviación típica de los 23 patrones se muestra en la figura 7.a. Resultan 12 autofunciones (figura 7.b) o Empirical Orthogonal Functions (EOF), las primeras de las cuales explican una gran parte de la varianza de los patrones (figura 7.c).

En la figura 8.a se muestra el campo Z500 del patrón 11 de Font «Anticiclón centroeuropeo», mostrado anteriormente, desplegando en la figura 8.b sus componentes principales en el espacio de las EOF calculadas previamente. Puede verse como las componentes 0 y 1 (tal vez también 4) «pesan» más que el resto, por lo que el patrón queda razonablemente bien explicado con 2 autofunciones. Los primeros intentos de agrupar los patrones de Font utilizando estas EOF arrojan resultados prometedores. En la figura 8.c puede verse un ejemplo, donde los ejes X e Y corresponden a las dimensiones 1 y 2. Resultan 8 grupos o clusters en diferentes colores. 
a)
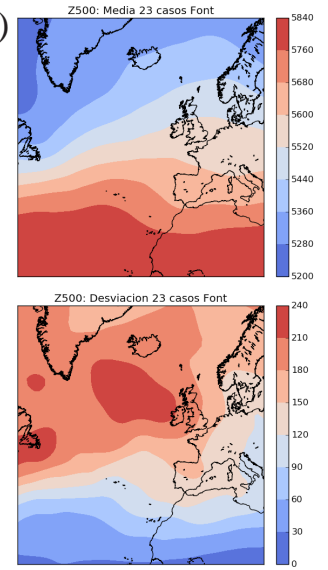

b)
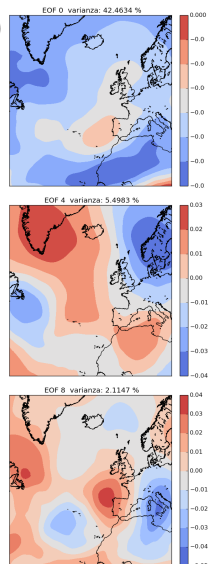
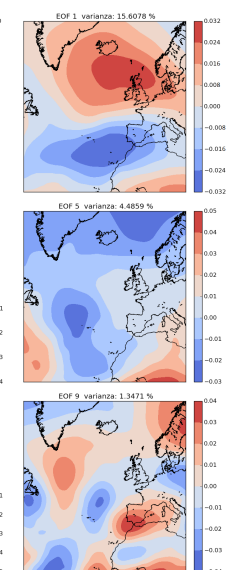
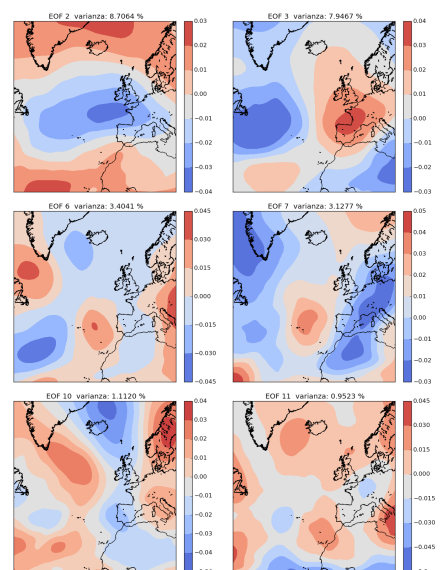

c)
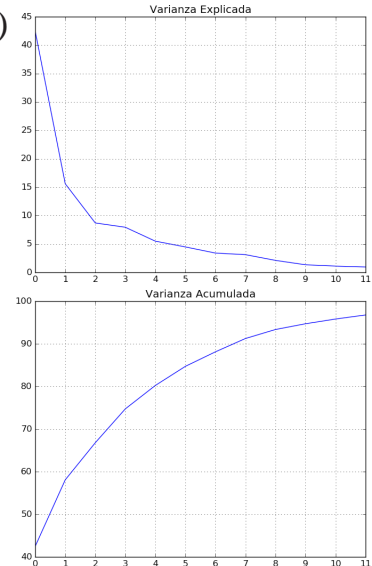

Figura 7. a) Media (arriba) y desviación típica (abajo) de los 23 patrones de Z500 de Font.

b) Las 12 autofunciones (EOF, numerados del 00 al 11) de los patrones Font aplicando la técnica PCA descrita en el texto. c) Varianza explicada (arriba) para cada autofunción y varianza acumulada correspondiente (abajo).

a)

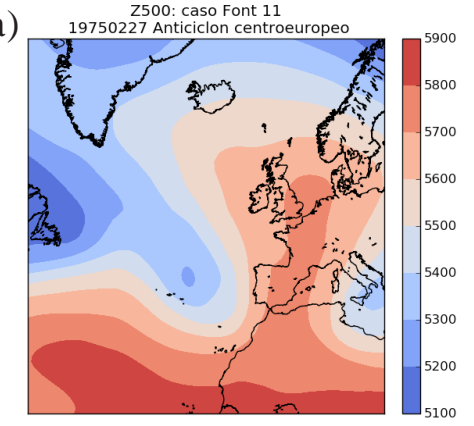

b)

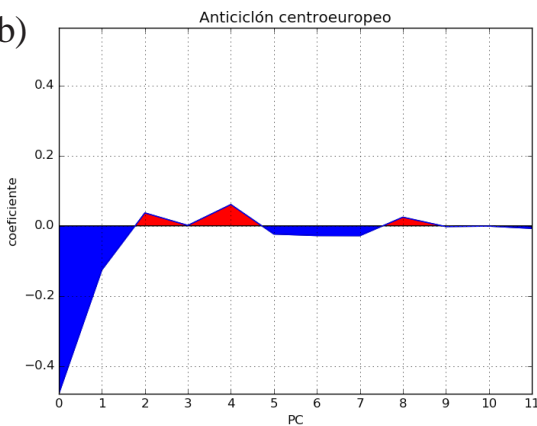

c)

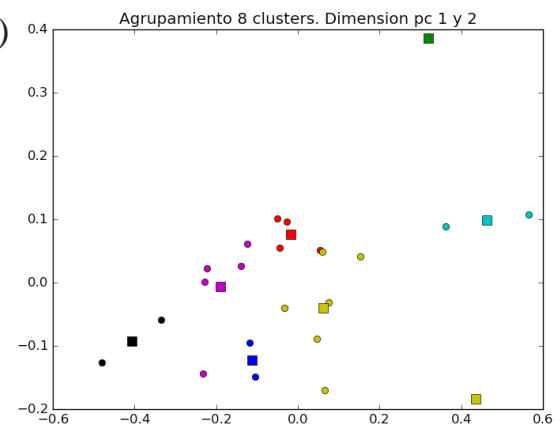

Figura 8. a) Campo Z500 del patrón 11 de Font «Anticiclón centroeuropeo», mostrado en las figuras 3 y 5.

b) Componentes principales de este patrón según los 12 EOF mostrados en la figura 8.b.

c) Dimensiones 1 y 2, según los mismos EOF, de un posible agrupamiento de los 23 patrones de Font, resultando 8 grupos o clusters.

\section{CONCLUSIONES}

Las clasificaciones sinópticas ayudan a entender la meteorología en esa escala mediante patrones tipo. En la actualidad, se hace necesaria una convergencia entre las clasificaciones objetivas (se muestra la de RiBALAYGUA y BorÉn, 1995, como ejemplo), basadas en modelos numéricos y algoritmos de agrupamiento y las clasificaciones subjetivas, basadas en modelos conceptuales (se muestra la de FonT, 2000, como ejemplo). En este estudio se recicla la clasificación de Font, recogiendo datos del reanálisis ERA40 del ECMWF correspondientes a los 23 patrones de Font y ploteándolos con técnicas y pautas modernas de visualización de campos para su uso en predicción operativa. Se ha aplicado, además, la técnica estadística de análisis de componentes principales (PCA, por sus siglas en inglés) explorando una simplificación opcional y un mejor entendimiento de los 23 patrones Font. Los primeros resultados apuntan a un espacio de 12 dimensiones, consiguiendo en ese espacio un conjunto de 8 grupos de patrones.

Este estudio, como punto de partida, plantea un abanico de líneas de investigación y aplicación. 1) El reciclado de conocimiento que supone el enriquecimiento visual de la clasificación de Font, así como la convergencia de enfoques (objetivo-subjetivo) encuentra aplicación directa en formación, divulgación y comunicación meteorológica, por lo que se sugiere su inclusión en cursos y su publicación en la web de AEMET. 2) La 
consistencia entre ambos enfoques (objetivo y subjetivo) puede explorarse ahora con mayor profundidad. 3) La aplicación de otras técnicas y parámetros de agrupamiento pueden arrojar evidencias de las que sacar partido. 4) Se pone así un granito de arena para ayudar a entender mejor la naturaleza de la variabilidad y diversidad de estos patrones.

\section{AGRADECIMIENTOS}

El personal de la Biblioteca y Reprografía de AEMET ayudó con el material gráfico más antiguo y difícil de conseguir.

\section{REFERENCIAS}

Borén Iglesias, R., Ribalaygua Batalla, J. y Balairón Ruiz, L., 1995. Método analógico de simulación de escenarios climáticos a escala comarcal. Informe N. 4 del Servicio de Análisis e Investigación del Clima, INM, Madrid.

Calvo, J., 1993. Clasificación de flujos en 500 hPa sobre la Península Ibérica. Nota técnica N. 32 del Servicio de Predicción Numérica, INM.

Fernández García, A. D. J., Martín Vide, J., Díaz Pérez, Y. A. y Mestre Barceló, A., 2003. Aplicación de los procesos sinópticos objetivos a la península ibérica en otoño. Investigaciones Geográficas (Esp), 31.

Font Tullot, I., 2000. Climatología de España y Portugal. Universidad de Salamanca.

Hartigan, J. A. y Hartigan, J. A., 1975. Clustering algorithms. New York: Wiley.

Holton, J. R. y Haкim, G. J., 2012. An introduction to dynamic meteorology. Academic press.

JoLLIFFE, I. T., 1986. Principal Component Analysis and Factor Analysis. En: Principal component analysis (pp. 115-128). Springer New York.

LINÉs, A., 1981. Perturbaciones típicas que afectan a la Península Ibérica y precipitaciones asociadas. INM, serie A, N. ${ }^{\circ} 80$, Madrid.

Mass, C. F., Edmon, H. J., Friedman, H. J., Cheney, N. R. y Recker, E. E., 1987. The use of compact discs for the storage of large meteorological and oceanographic data sets. Bulletin of the American Meteorological Society, 68 (2), 1556-1558.

Peña, J. C., Aran, M., Pérez Zanón, N., Casas Castillo, M., Rodríguez Solá, R. y Redaño Xipell, A., 2015. Análisis de las situaciones sinópticas correspondientes a episodios de lluvia severa en Barcelona. En: XXXV Reunión Bienal de la Real Sociedad Española de Física. Libro de Resúmenes (pp. 450-451). Real Sociedad Española de Física (RSEF).

Persson, A., 2001. User Guide to ECMWF forecast products. ECMWF.

Petisco, E. y Martín, J. M., 1995. Caracterización de la circulación general atmosférica en la Península Ibérica y Baleares. Nota técnica 5 del Servicio de Análisis e Investigación del Clima, INM, Madrid.

Ribalaygua Batalla, J. y Borén Iglesias, R., 1995. Clasificación de patrones espaciales de precipitación diaria sobre la España peninsular y Baleárica. Informe N. 3 del Servicio de Análisis e Investigación del Clima, INM, Madrid. 
SÁNCHEZ RodRíGuEZ, J., 1993. Situaciones atmosféricas en España. INM. Madrid.

Ribalaygua Batalla, J., Borén Iglesias, R. y Balairón Ruiz, L., 1999. Clasificación de repartos de precipitación diaria sobre la España peninsular y baleárica: aplicación para la generación de clasificaciones atmosféricas de apoyo a la predicción de precipitaciones.

Soriano Ortiz, C., Fernández, A. G. y Martín Vide, J., 2003. Sobre el uso de los procesos sinópticos objetivos y los modelos meteorológicos a alta resolución para el estudio de los patrones circulatorios a escala regional.

Strahler, A. y Strahler, A., 2007. Physical geography. John Wiley \& Sons.

Uppala, S. M., Kållberg, P. W., Simmons, A. J., Andrae, U., Bechtold, V. D., Fiorino, M., ... y Li, X., 2005. The ERA40 reanalysis. Quarterly Journal of the royal meteorological society, 131 (612), 2961-3012. 